\title{
Búsqueda in silico de análogos de cefalosporina con potencial actividad antibiótica dual.
}

\author{
In Silico search for cephalosporin analogues with potential dual antibiotic \\ activity.
}

\section{Maicol Ahumedo Monterrosa ${ }^{1,2^{*}}$, Jorge Anaya Gil ${ }^{2}$, Wilson Maldonado Rojas ${ }^{3}$}

${ }^{1}$ Profesor, Facultad de ingenierías, Grupo CIPTEC, Fundación Universitaria Tecnológico Comfenalco, Cll 30 N 30D-60, Cartagena, Colombia.

mahumedo@tecnocomfenalco.edu.co

2 Profesor, Facultad de Ciencias Farmacéuticas, Grupo de Productos Naturales, Universidad de Cartagena, Campus de Zaragocilla, Cartagena, Colombia.

janayag@unicartagena.edu.co

3 Profesor, Facultad de Ciencias Farmacéuticas, Grupo de Química Ambiental y computacional, Universidad de Cartagena, Campus de Zaragocilla, Cartagena, Colombia.

wmaldonador@unicartagena.edu.co

Recibido: 15/ago/2021 Revisado: 30/sep/2021

Aceptado: 30/oct/2021 Publicado: 30/dic/2021

\begin{abstract}
Resumen El surgimiento y propagación de bacterias patógenas multirresistentes se ha convertido en un problema de salud pública debido al aumento de casos en los cuales no se cuenta con un tratamiento adecuado; por lo que la búsqueda de compuestos con propiedades antibióticas contra bacterias resistentes a los antibióticos tradicionales se ha vuelto prioritaria. En este trabajo se realizó una búsqueda in silico de compuestos análogos de cefalosporinas y se estudió su interacción con las enzimas transpeptidasas y betalactamasas de Escherichia coli. Para lo cual se descargaron de la base de datos PubChem 163 moléculas; las afinidades de estas fueron evaluadas por acoplamiento molecular con las estructuras de transpeptidasa y betalactamasa usando el programa AutoDock v4.2. Se identificaron nueve compuestos con mayor energía de afinidad por la transpeptidasa y menor afinidad por la betalactamasa; a dos de los cuales les fueron realizadas modificaciones químicas in silico con el propósito de evaluar el efecto de los sustituyentes en los valores de afinidad, así como sus propiedades ADME. La cefaperozona monobactam modificada con un grupo metilo presentó una mejor afinidad por la transpeptidasa (de -6.8 a $-7.8 \mathrm{kcal} / \mathrm{mol}$ ) y disminuyó la afinidad por la betalactamasa (-5.9 a -5.8 $\mathrm{kcal} / \mathrm{mol}$ ) lo que demostró el potencial dual de esta molécula.
\end{abstract}

Palabras claves Cefalosporina; in silico; Escherichia coli; transpeptidasa; betalactamasa.

Abstract The emergence and spread of multidrug-resistant pathogenic bacteria has become a public health problem due to the increase in the number of cases for which there is no adequate treatment; therefore, the search for compounds with antibiotic properties against bacteria resistant to traditional antibiotics has become a priority. In this work we performed an in silico search for cephalosporin analogues and studied their interaction with Escherichia coli transpeptidases and beta lactamases. For this purpose, 163 molecules were downloaded from the PubChem database; their affinity was evaluated by molecular docking with the transpeptidase and beta-lactamase structures using the AutoDock v4.2 program. Nine compounds with higher affinity energy for transpeptidase and lower affinity for beta-lactamase were identified; two of them were modified in order to evaluate the effect of the substituents on the affinity values and their ADME properties. Cefaperozone monobactam modified with a methyl group presented a better affinity for transpeptidase (from -6.8 to -7.8 $\mathrm{kcal} / \mathrm{mol})$ and decreased affinity for beta-lactamase $(-5.9$ to $-5.8 \mathrm{kcal} / \mathrm{mol})$, which demonstrated the dual potential of this molecule.

Keywords Cephalosporin; in silico; Escherichia coli; transpeptidase; beta-lactamase.

Teknos Revista Científica. | Volumen 21 No.2 - diciembre 2021 | ISSN 1900-7388 (papel) | ISSN 2539-2190 (digital)

DOI: https://doi.org/10.25044/issn.2539-2190 


\section{Introducción}

Durante los últimos años, el tratamiento de las enfermedades infecciosas ha provocado un aumento en la resistencia de microorganismos a una variedad de moléculas con actividad antibiótica, perteneciente a diferentes grupos (Acar et al., 2019). Los antibióticos betalactámicos donde se encuentra la familia de las cefalosporinas, han demostrado su actividad antibiótica, actuando principalmente sobre las membranas de las bacterias, inhibiendo la acción transpeptidasas, provocando debilidad en su pared celular, desencadenando eventos que conllevan a su muerte (Hincapié et al, 2021).

Es importante resaltar que algunos antibióticos derivados de compuestos betalactámicos generalmente son hidrolizados en medio ácido, provocando la degradación del anillo betalactámico, lo que conduce a la pérdida de la actividad antibiótica de estos compuestos (Acevedo et al., 2021). El proceso anterior, está estrechamente relacionado con infecciones causadas por microorganismos, asociados con estancias hospitalarias prolongadas, mayores tasas de fracaso terapéutico, aumento en la mortalidad, y un incremento en los costos derivados de la atención clínica que amenaza la sostenibilidad de cualquier sistema de salud (Hernandez-Gómez et al., 2014); adicionalmente en países en vía de desarrollo, muchas personas abusan del uso de antibióticos, llegando al punto de automedicarse; circunstancias que han propiciado la aparición de microorganismos resistentes al tratamiento con antibióticos. El aumento de organismos cada vez resistentes a los antibióticos y la pérdida de actividad antimicrobiana de los compuestos diseñados para este fin, establecen nuevos retos direccionados a la búsqueda de estrategias para el tratamiento de infecciones resistentes a los antibióticos (Owen et al., 2017).

La bacteria Escherichia coli (E. coli) es una de las principales bacterias multirresistentes involucradas en infecciones nosocomiales y comunitarias que con mayor frecuencia se ha ido diseminando, y por lo tanto, aumentando la generación de nuevas cepas y cambios epidemiológicos (García-Hernández et al., 2011); determinándose que dicha bacteria es resistente a antibióticos tipo cefalosporinas por la producción de enzimas betalactamasa de espectro extendido (BLEE), principal mecanismo que confiere resistencia a las cefalosporinas (García et al., 2012). Hecho demostrado por el aumento de infecciones por cepas de E. coli resistentes a cefalosporinas en diversas zonas de Latinoamérica (OMS 2014), categorizándola como una de las regiones del mundo con mayor prevalencia en producción de BLEE por enterobacterias con tasas de un $8.5 \%$ a $18.1 \%$ para $E$. coli, en comparación con Estados Unidos y Europa (Abreu et al., 2014); incluyendo a Colombia, como uno de los países con alta prevalencia de microorganismos productores de BLEE (Red Nacional de Vigilancia 2010).

Teniendo en cuenta lo expuesto anteriormente, se hace evidente que existe un alto grado de resistencia antibiótica a compuestos tipo cefalosporina (UrbinaDaza et al., 2019), por lo que es urgente la necesidad de implementar en los tratamientos farmacoterapéuticos; nuevos compuestos que supriman todos estos inconvenientes y sean eficaces contra estos microrganismos patógenos resistentes. Por lo tanto, se requieren estrategias que permitan encontrar nuevas moléculas con actividad bactericida, las cuales sean resistentes a los mecanismos de defensa de las bacterias. Actualmente las herramientas basadas en química computacional, han surgido como una alternativa para la búsqueda inicial de compuestos promisorios, basados en cribado virtual de librerías y bases de datos (Yildirim et al., 2017; Mahasenan et al., 2017). En la presente investigación se propuso identificar y diseñar por medio de modificaciones in silico, compuestos análogos de la cefalosporina que presenten mayor afinidad hacia transpeptidasa y menor afinidad por la betalactamasa del $E$. coli, con el fin de identificar compuestos con posible actividad antibiótica dual. 


\section{Metodología}

\subsection{Búsqueda de los compuestos análogos de cefalosporina en la base de datos PubChem.}

Se realizó la búsqueda de las estructuras químicas de compuestos análogos del farmacóforo de la cefalosporina, en la base de datos PubChem (http://pubchem.ncbi.nlm.nih.gov); las moléculas seleccionadas se descargaron con formato sdf y con el programa GaussView 5.0 (Dennington et al., 2009) fueron optimizadas geométricamente, utilizando el método semiempírico PM6 (Mendoza-Huizar, 2018). Posteriormente las estructuras optimizadas fueron almacenadas en formato PDBQT, para la posterior evaluación de la afinidad teórica por acoplamiento molecular proteína-ligando.

\subsection{Estudio de la interacción de las diferentes moléculas descargadas en el sitio activo de la transpeptidasa de $E$. coli utilizando acoplamiento molecular.}

\subsubsection{Preparación para el acoplamiento molecular}

Para realizar la validación de los protocolos de acoplamiento molecular en el sistema modelado, se utilizó el programa AutoDock v4.2 (Morris et al, 2009). El procedimiento para esta validación fue el descrito a continuación:

\subsubsection{Preparación de las estructuras de la transpeptidasa y ligandos.}

De la base de datos PDB (Protein Data Bank) se descargó la estructura $3 \mathrm{D}$ de la proteína transpeptidasa de una cepa de E. coli, con ID 3MZE en forma de complejo cocristalizado con el ligando nativo cefoxitina con ID DB01331 del DrugBank, el cual fue empleado para la validación de los cálculos de acoplamiento molecular. Teniendo en cuenta que dicho ligando nativo es un análogo estructural de la molécula de referencia (cefotaxima).

La proteína fue visualizada, permitiendo la identificación del ligando nativo en el sitio activo, usando el programa SYBYL-X v2.1.1 (Tripos 2013), de la cual se extrajo la estructura del ligando nativo (cocristalizado), se eliminaron las moléculas de agua y cofactores con los cuales viene resuelta la proteína cristalizada. Posteriormente se adicionaron todos los hidrógenos a la proteína y se asignaron cargas de Gasteiger-Marsilli (Kumar et al., 2014).

Por otra parte, el ligando nativo fue preparado en AutoDocktools v1.5.6 (Morris et al., 2009), donde se adicionaron los hidrógenos polares, se identificaron los enlaces rotables y por último se adicionaron las cargas de Gasteiger-Marsilli. Los archivos resultantes fueron almacenados en formato PDBQT, para los cálculos de acoplamiento molecular proteína-ligando.

\subsubsection{Acoplamiento molecular}

Los parámetros del acoplamiento proteína-ligando con AutoDock v4.2 fueron: caja del grid centrada en el sitio de unión del ligando nativo $(X=42.86 \mathrm{Y}=6.19$ y $Z=$ 30.49 ) con un tamaño $51 \times 51 \times 51 \AA$. Para la búsqueda conformacional fue seleccionado el algoritmo genético Lamarckian (Morris et al., 1998), estipulando 100 corridas como el número máximo de conformaciones generadas. Los demás parámetros de tomaron del artículo de Ahumedo (2019).

\subsubsection{Selección de la mejor conformación e identificación de las interacciones proteína-ligando}

Las mejores conformaciones escogidas para las moléculas se visualizaron con el programa MOE, dando importancia a la ubicación del grupo farmacofórico en el sitio activo. 


\subsection{Modificación química de las mejores moléculas con posible actividad antibiótica dual}

La molécula que presentó mayor valor de afinidad por la enzima transpeptidasa y menor afinidad respecto a la enzima betalactamasa, fue sometida a un cambio en su estructura química; se le agregó un grupo metilo para estudiar el efecto de la sustitución en los valores de energía de afinidad.

\subsection{Predicción de las propiedades ADME para compuestos modificados}

Con el fin de determinar el posible comportamiento de las moléculas en los procesos biológicos de adsorción, distribución, metabolismo y excreción, se determinaron algunos parámetros necesarios para realizar las predicciones de las propiedades ADME del compuesto que presentó mayor afinidad a la transpeptidasa y menor afinidad a la enzima betalactamasa, así como a las moléculas que fueron modificadas químicamente; los parámetros evaluados corresponden al peso molecular coeficiente de reparto octanol-agua $(\log \mathrm{P})$, número de átomos donadores de hidrógeno (HBD), número de átomos aceptores de hidrógeno (HBA), número de enlaces rotativos y Área de Superficie Polar Topológica (TPSA por sus siglas en inglés). Esta predicción fue realizada con el servidor web swissADME (Daina et al., 2017).

\section{Resultados y discusión}

\subsection{Selección de compuestos análogos de cefalosporina en la base de datos de PubChem}

Al realizar la búsqueda de compuestos análogos de cefalosporina en la base de datos de PubChem (http://pubchem.ncbi.nlm.nih.gov), se utilizó como filtro el anillo betalactamico de la cefalosporina, para identificar los análogos que presentan en su estructura el farmacóforo de este tipo de molécula, es decir, se escogieron compuestos que contuvieran en su estructura un anillo betalactámico; 163 moléculas cumplían con los parámetros de búsqueda, a las cuales se les comparó la energía de afinidad por la transpeptidasa y la betalactamasa respecto a la molécula de cefotaxima (molécula de referencia), realizando cálculos de acoplamiento molecular con el programa Autodock 4.2; aquellos compuestos con igual o mayor afinidad por la transpeptidasa o igual o menor afinidad por la betalactamasa que el compuesto de referencia, se seleccionaron para realizar el estudio de relación estructura - actividad. En la tabla 1 se muestran los 9 compuestos con valores energéticos que pasaron el filtro de afinidad.

\subsection{Validación de la metodología propuesta para evaluación de la interacción de moléculas evaluadas con las enzimas transpeptidasa y betalactamasa.}

Con el objetivo de validar los protocolos de acoplamiento molecular utilizado en el presente trabajo, fue utilizado el programa de acoplamiento molecular, AutoDock v4.2 (Morris et al, 2009). Para realizar la validación con el programa, se comparó la estructura cocristalizada del ligando nativo (cefoxitina con ID DB01331 del DrugBank) versus su estructura acoplada en el sitio activo del receptor transpeptidasa (Código PDB 3MZE). Los parámetros de validación empleados fueron el criterio visual y el criterio de desviación estándar cuadrática media (RMSD), el cual mide la divergencia de dos estructuras entre los pares de átomos alineados después de la superposición óptima, pero que se ve fuertemente influenciada por regiones de mucha variabilidad. Valores de RMSD por debajo de $2.0 \AA$; son considerados correctos. (Pagadala et al., 2017).

En la figura 1 se muestra el alineamiento entre la conformación del ligando nativo correspondiente al complejo cocristalizado en color verde y la conformación resultante del acoplamiento mostrada en color rojo. Siguiendo el criterio visual, se observa que la conformación predicha está ubicada en el sitio de unión de la transpeptidasa tomando una conformación semejante a la que presenta el ligando 
nativo en su estructura cristalizada. Ahora, según el criterio RMSD, se obtuvo un valor de $1.12 \AA$ para la conformación de menor energía de unión, considerándose como una conformación correcta, por lo cual se puede afirmar que AutoDock v4.2 reproduce adecuadamente el modo de unión y las interacciones entre la transpeptidasa y su ligando nativo, lo que brinda certeza acerca de las predicciones que dicho programa pueda realizar sobre el conjunto de moléculas seleccionadas.

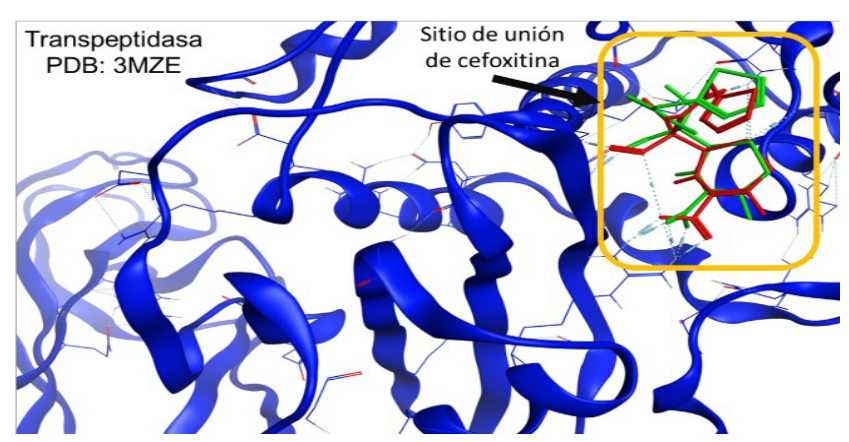

Figura 1. Alineamiento entre el ligando nativo presente en el complejo cocristalizado cefoxitina-transpeptidasa (en color verde) y el ligando nativo resultante del acoplamiento molecular (en color rojo), utilizando el programa AutoDock v4.2.

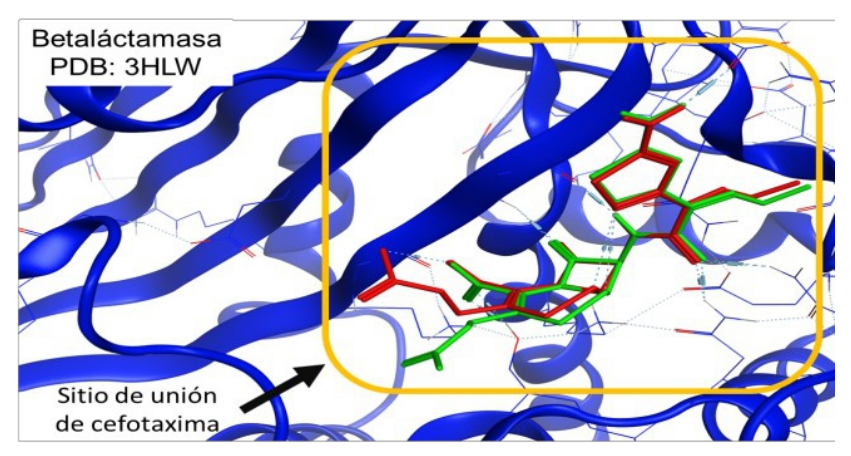

Figura 2. Alineamiento entre el ligando nativo presente en el complejo cocristalizado cefotaxima-betaláctamasa (en color verde) y el ligando nativo resultante del acoplamiento molecular (en color rojo), utilizando el programa AutoDock v4.2.

De manera análoga, se realizó la validación de la metodología de acoplamiento molecular para la enzima betaláctamasa. Se comparó la estructura cocristalizada del ligando nativo (cefotaxima con ID
DB00493 del DrugBank) versus su estructura acoplada en el sitio activo del receptor betaláctamasa (Código PDB: 3HLW). La figura 2 muestra el alineamiento entre la conformación del ligando nativo correspondiente al complejo cocristalizado en color verde y la conformación resultante del acoplamiento mostrada en color rojo, que según el criterio visual, esta última adopta una conformación semejante a la conformación del ligando nativo, logrando observar que las cadenas sustituyentes del anillo betalactámico muestran variabilidad conformacional y por tanto contribuyen en mayor medida a la divergencia de las estructuras, obteniéndose un valor de RMSD de 1.05 $\AA$, el cual es considerado correcto.

\subsection{Acoplamiento molecular entre las diferentes moléculas seleccionadas y las proteínas transpeptidasa y betalactamasa de E. coli}

Para estudiar las interacciones entre las diferentes moléculas seleccionadas y el sitio activo del receptor se seleccionó la conformación que reportó la menor energía de unión, entre todas las conformaciones obtenidas para cada ligando. Asimismo, para las conformaciones seleccionadas se tuvo en cuenta la orientación del grupo farmacofórico en el sitio activo del receptor, tomando como referencia la orientación del ligando nativo. Los valores de energías de unión de la enzima con la mejor conformación de cada ligando obtenidos a partir del proceso de acoplamiento molecular entre la enzima transpeptidasa (Código PDB: 3MZE) y las 9 moléculas seleccionadas, así como los valores de energías de unión de la enzima con la mejor conformación de cada ligando obtenidos a partir de la evaluación por acoplamiento molecular entre la enzima betaláctamasa (Código PDB 3HLW) y las moléculas seleccionadas se muestran en la tabla 1.

Después de ejecutados los protocolos de acoplamiento molecular proteína-ligando. Se encontró que nueve moléculas presentan características promisorias como posible antibiótico dual, es decir, se identificaron moléculas que presentaron mayor 
energía de unión a la transpeptidasa que la cefotaxima (molécula de referencia) y menor energía de unión a la betalactamasa si se compara igualmente con el compuesto de referencia; las nueve moléculas seleccionadas fueron: cefoperazona, cefoperazona monobactam, cefotetan, cefalosporina mustard, cefempidona, cefmepidium, cefalosporina 87/312, cefoselis y ceftarolina fosamil; estas moléculas presentaron características comunes tales como los residuos de aminoácidos serinicos de la transpeptidasa, que tienen la posibilidad de formar enlaces covalentes con este tipo de moléculas al estar cerca del farmacóforo, mientras que la hidrolisis por parte de la betalactamasa se veía impedida en algunos casos por efectos estéricos y en otros casos por interacciones intermoleculares e intramoleculares que provocaban la disminución de la afinidad de esta enzima por el ligando, lo que impide que se presente el fenómeno de resistencia bacteriana o por lo menos sea más difícil que aparezca por este mecanismo. A continuación, se presentan las interacciones entre la molécula que presentó mayor afinidad por la transpeptidasa (cefoperazona monobactam) y el compuesto que presentó menor energía de afinidad por la betalactamasa (Cefalosporina 87/312).

Tabla 1. Afinidad teórica entre las moléculas seleccionadas y las enzimas transpeptidasa y betalactamasa.

\begin{tabular}{|c|c|c|c|}
\hline No. & Compuesto & $\begin{array}{c}\text { Afinidad } \\
\text { Transpeptidasa } \\
(\mathbf{k c a l} / \mathbf{m o l})\end{array}$ & $\begin{array}{c}\text { Afinidad } \\
\text { Betaláctamasa } \\
(\mathbf{k c a l} / \mathbf{m o l})\end{array}$ \\
\hline 0 & Cefotaxima & -6.1 & -6.2 \\
\hline 1 & Cefoselis & -6.2 & -6.2 \\
\hline 2 & $\begin{array}{c}\text { Ceftarolina } \\
\text { fosamil }\end{array}$ & -6.4 & -6.2 \\
\hline 3 & $\begin{array}{c}\text { Cefoperazona } \\
\text { monobactam }\end{array}$ & -6.8 & -5.9 \\
\hline 4 & Cefotetan & -6.2 & -5.9 \\
\hline 5 & $\begin{array}{c}\text { Cefalosporina } \\
\text { mustard }\end{array}$ & -6.3 & -5.4 \\
\hline 6 & Cefempidona & -6.6 & -5.3 \\
\hline 7 & Cefoperazona & -6.7 & -4.6 \\
\hline 8 & $\begin{array}{c}\text { Cefalosporina } \\
87 / 312\end{array}$ & -6.5 & -3.6 \\
\hline 9 & Cefmepidium & -6.5 & -5.3 \\
\hline
\end{tabular}

En la figura 3 se representa el acoplamiento entre la cefoperazona monobactam (CID 135784) (este compuesto no presenta anillo dihidrotiazinico) y el sitio activo de la transpeptidasa. Se observa que los aminoácidos Ala 43, His 216 y Ser 44 interactúan con el anillo lactámico y otros residuos lo hacen con el grupo bisulfato que está adyacente a la betalactama estabilizando así la molécula; por lo que su energía de afinidad es superior a la cefotoxima en las mismas condiciones.

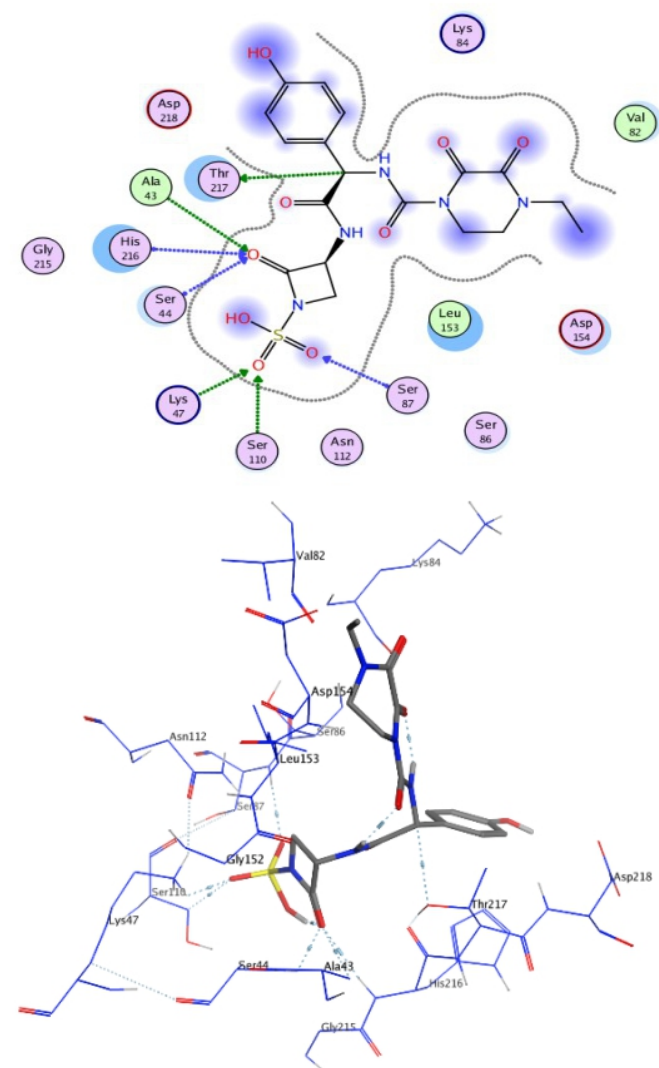

Figura 3. Interacciones presentes entre la cefoperazona monobactam y el sitio activo de la transpeptidasa en $2 \mathrm{D}$ y 3D.

El acoplamiento molecular entre cefoperazona monobactam y el sitio activo de la betalactamasa, se representan en la figura 4 , observándose que el farmacóforo no está interaccionando directamente con ninguno de los aminoácidos del sitio catalítico, pero otras partes de la molécula forman interacciones tipo 
puentes de hidrógeno y de Van der Waals, que en determinado instante permitirán que la molécula reaccione y se hidrolice el anillo lactámico, perdiendo así su capacidad antibiótica.
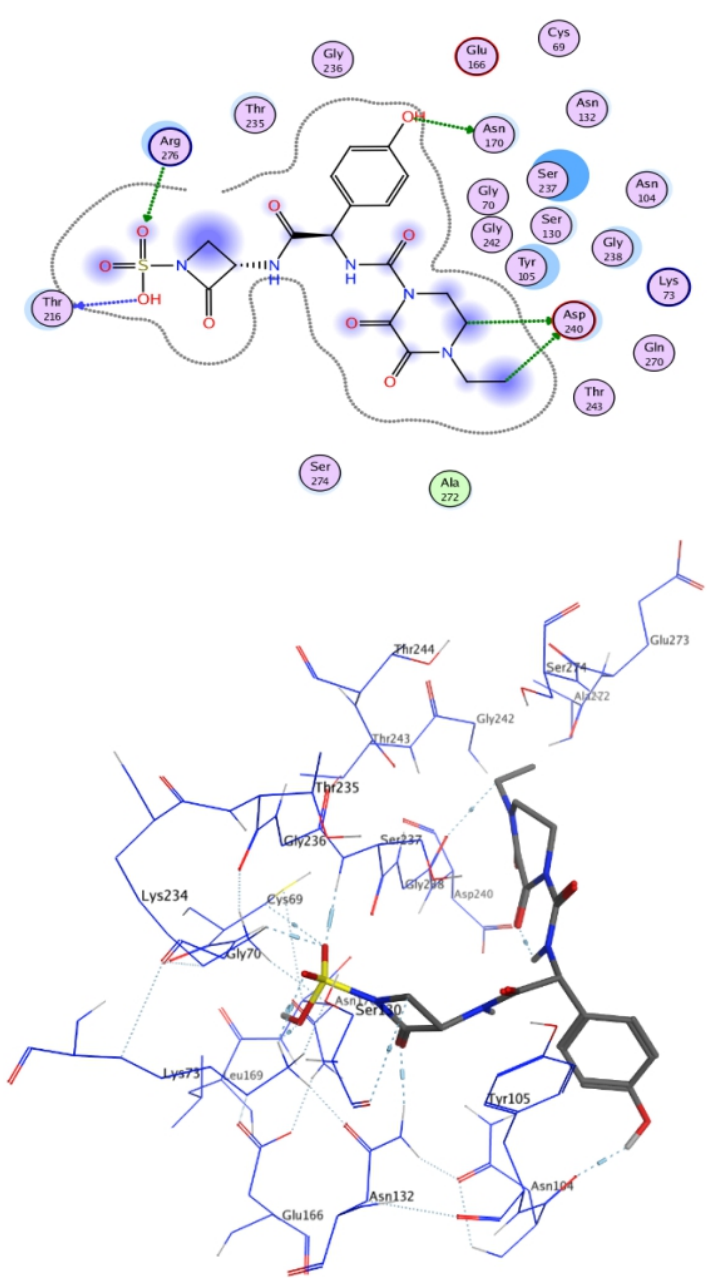

Figura 4. Interacciones presentes entre la cefoperazona monobactam y el sitio activo de la betalactamasa en 2D y 3D.

La molécula de cefalosporina 87/312 (CID 44123521) presenta afinidad favorable por la transpeptidasa, con respecto a la molécula de referencia, lo cual puede explicarse, de acuerdo con la figura 5 a la interacción entre Ser 87 y el anillo betalactámico, así como la Ser 110 interactúa con el azufre del anillo dihidrotiazinico y el grupo carbonilo; y la estabilidad que le ofrece residuos como Arg 198 al estabilizar el grupo nitro, así como la His 216 al estabilizar el anillo de tiofeno.
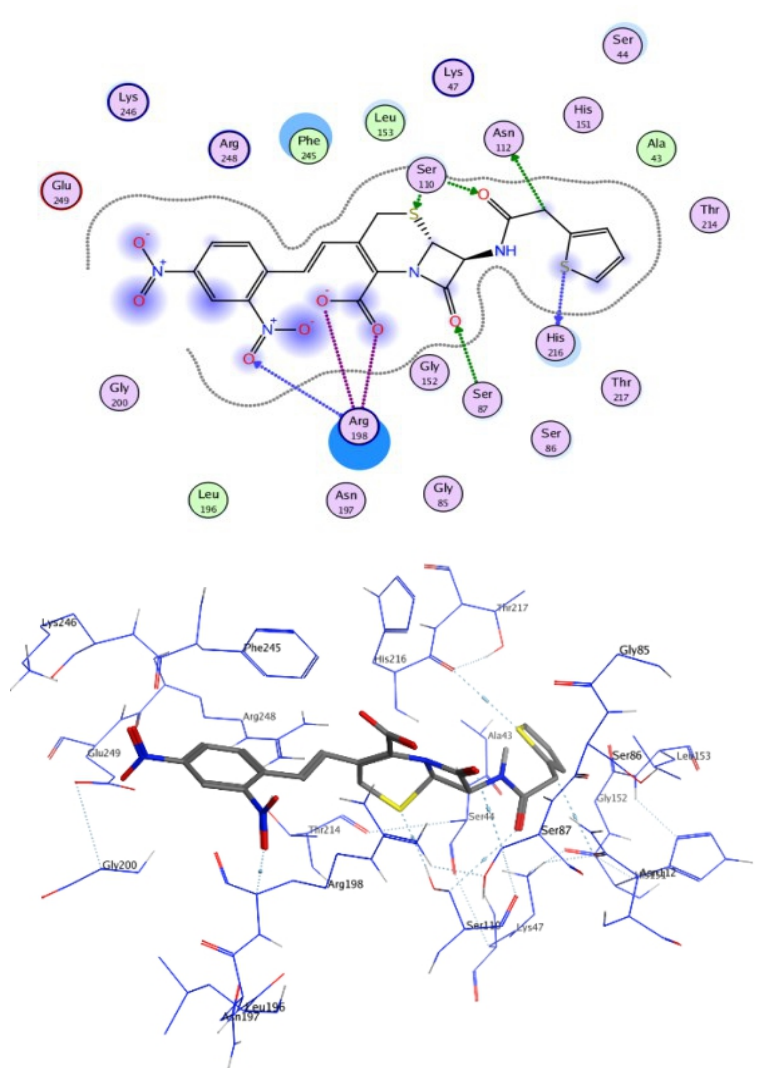

Figura 5. Interacciones presentes entre la cefalosporina $87 / 312$ y el sitio activo de la transpeptidasa en $2 \mathrm{D}$ y $3 \mathrm{D}$.

La molécula de cefalosporina $87 / 312$ es la que presenta la menor afinidad por la enzima betalactamasa. Lo que podría inferirse que es un potencial candidato como fármaco resistente a betalactamasa; de acuerdo a la figura 6 , el grupo carbonil adyacente al farmacóforo que interacciona con Asn 123 y los grupos nitros que interactúan con Arg 276 y los grupos voluminosos como el tiofeno y el nitrofenilo pueden inducir a la resistencia a un ataque nucleofílico debido a impedimentos estérico.

Adicionalmente en la presente investigación se decidió realizar una adición de un grupo metilo a la molécula que presentó mayor afinidad por la transpeptidasa (cefoperazona monobactan) y al compuesto que presentó menor afinidad por la betalactamasa (cefalosporina 87/312) con el fin de evaluar el efecto 
de esa sustitución en los valores de afinidad y las propiedades ADME. Las discusiones relacionadas con este análisis son tratadas en los siguientes apartados.

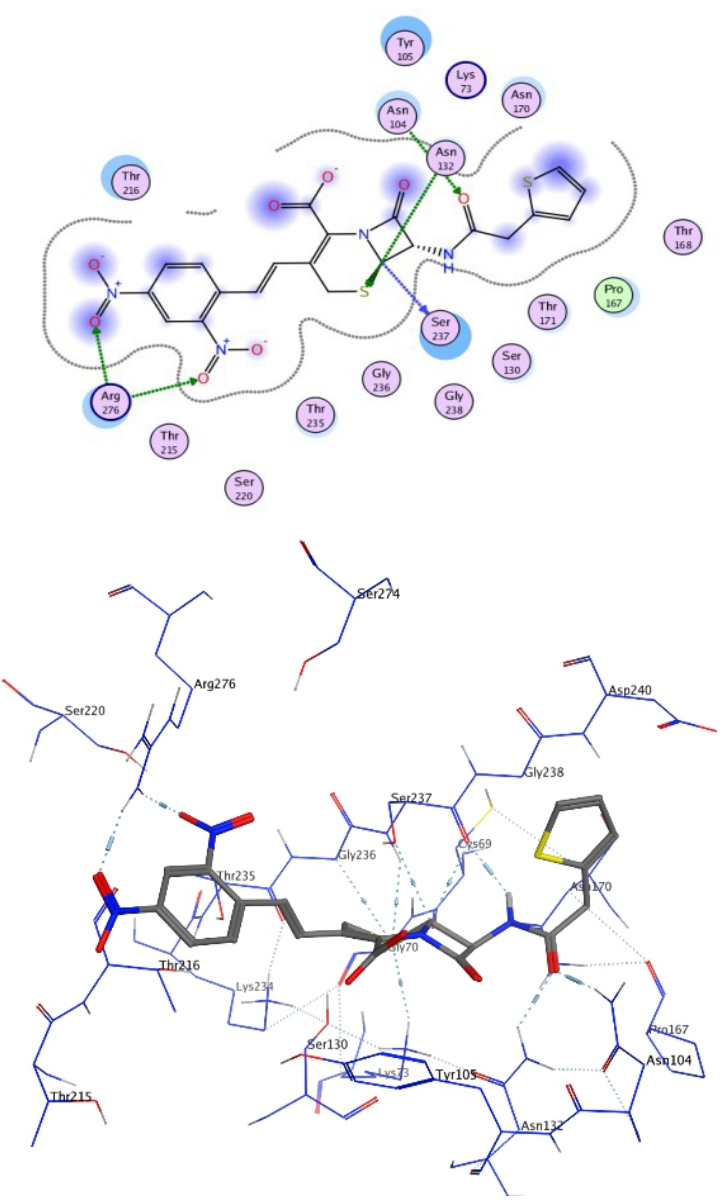

Figura 6. Interacciones presentes entre la cefalosporina $87 / 312$ y el sitio activo de la betalactamasa en $2 \mathrm{D}$.

\subsection{Modificación química de las mejores moléculas con posible actividad antibiotica dual}

Como se mencionó anteriormente se le adicionó un grupo metilo a las estructuras originales de un par de moléculas para evaluar el efecto de la sustitución. Este grupo fue seleccionado debido al carácter lipofilico del mismo y puede provocar efectos inductivos de electrones (Kapoor \& Kumar, 2019) y no se escogió grupos alquílicos más grandes para evitar problemas por efectos estéricos. Para el efecto de diferenciación, los nuevos compuestos se denominaron metil cefoperazona monobactam y metil cefalosporina $87 / 312$. En la tabla 2 se hace comparativo entre las energías de afinidad originalmente calculadas y los valores hallados luego de la sustitución.

De acuerdo a la tabla 2, la adición del grupo metilo en el compuesto cefoperazona monobactam mejoró notablemente la energía de afinidad por la enzima transpeptidasa (de -6.8 a $-7.8 \mathrm{kcal} / \mathrm{mol}$ ) y disminuyó en $0.1 \mathrm{kcal} / \mathrm{mol}$, la afinidad por la betalactamasa. Esto indica que la adición del grupo metil en la estructura química mejora sus características como un potencial antibiótico dual.

Tabla 2. Valores de energía de afinidad, calculados para las moléculas sustituidas con el grupo metil y las estructuras originales.

\begin{tabular}{|c|c|c|}
\hline Compuesto & $\begin{array}{c}\text { Afinidad } \\
\text { Transpeptidasa } \\
\text { (kcal/mol) }\end{array}$ & $\begin{array}{c}\text { Afinidad } \\
\text { Betalactamasa } \\
\text { (kcal/mol) }\end{array}$ \\
\hline $\begin{array}{c}\text { Cefoperazona } \\
\text { monobactam }\end{array}$ & -6.8 & -5.9 \\
\hline $\begin{array}{c}\text { Metil } \\
\text { Cefoperazona } \\
\text { monobactam* }\end{array}$ & -7.8 & -5.8 \\
\hline $\begin{array}{c}\text { Cefalosporina } \\
87 / 312\end{array}$ & -6.5 & -3.6 \\
\hline $\begin{array}{c}\text { Metil } \\
\text { Cefalosporina } \\
87 / 312^{*}\end{array}$ & -6.6 & -4.1 \\
\hline
\end{tabular}

*compuesto modificado por adición de un grupo metil.

El otro compuesto que se estudió fue el metil cefalosporina 87/312, el cual de acuerdo a la tabla 2 , se observa que la sustitución con el grupo metilo provoca un aumento del $13.9 \%$ en la energía de afinidad por la enzima betalactamasa, lo cual indica que la modificación estructural logró un efecto no deseado; pues potencialmente está facilitando la lisis del farmacóforo, lo que conllevaría a una pérdida de la actividad bactericida. 


\subsection{Predicción de las propiedades ADME para compuestos modificados}

En la industria farmacéutica muchos compuestos que son candidatos como promisorios fármacos, son desechados por la carencia de propiedades farmacocinéticas óptimas, por lo que la predicción de las propiedades ADME juega un papel importante en el proceso de diseño de nuevos fármacos; por esta razón en la tabla 3 se muestran los resultados de la determinación de las propiedades fisicoquímicas de las moléculas evaluadas.

Tabla 3. Predicción de propiedades ADME de los compuestos modificados y sus moléculas de origen del servidor SwissADME.

\begin{tabular}{|c|c|c|c|c|}
\hline $\begin{array}{c}\text { Compue } \\
\text { sto }\end{array}$ & $\begin{array}{c}\text { Cefopera } \\
\text { zona } \\
\text { monobact } \\
\text { am }\end{array}$ & $\begin{array}{c}\text { Metil } \\
\text { cefoperaz } \\
\text { ona } \\
\text { monobac } \\
\text { tam }\end{array}$ & $\begin{array}{c}\text { Cefalospo } \\
\text { rina } \\
\mathbf{8 7 / 3 1 2}\end{array}$ & $\begin{array}{c}\text { Metil } \\
\text { cefalospo } \\
\text { rina } \\
\mathbf{8 7 / 3 1 2}\end{array}$ \\
\hline $\begin{array}{c}\text { Peso } \\
\text { molecu } \\
\text { lar } \\
\text { (g/mol) }\end{array}$ & 483.25 & 497.48 & 515.5 & 529.53 \\
\hline $\begin{array}{c}\text { Consen } \\
\text { so } \\
\text { LogP }\end{array}$ & -1.35 & -1.06 & 0.77 & 0.19 \\
\hline $\begin{array}{c}\text { HBD } \\
\text { HBA }\end{array}$ & 9 & 4 & 1 & 1 \\
\hline $\begin{array}{c}\text { Enlaces } \\
\text { rotativo } \\
\text { s }\end{array}$ & 9 & 9 & 9 & 8 \\
\hline $\begin{array}{c}\text { TPSA } \\
\text { (A }{ }^{2} \text { ) }\end{array}$ & 202.11 & 202.11 & 234.72 & 234.49 \\
\hline
\end{tabular}

Los parámetros mostrados corresponden a propiedades relevantes que influyen en la farmacocinética de una molécula. Las primeras cuatro filas por ejemplo, abarcan los criterios evaluados en la regla de Lipinski; la cual establece que una buena absorción o permeación, es más probable cuando la molécula tiene menos de 5 átomos donadores de hidrógeno (HBD), menos de 10 átomos aceptores de hidrógeno (HBA), un peso molecular inferior a 500
Dalton y un Log $\mathrm{P}$ calculado menor de 5; si 2 o más de estas condiciones no se cumplen, se espera que la molécula tenga problemas de absorción (Santos et al., 2018). Al aplicar la regla de Lipinski se observa que todos cumplen con las condiciones que son consideradas necesarias para una buena solubilidad en agua y permeabilidad intestinal; aunque cabe indicar que la cefalosporina $87 / 312$ y la metil cefalosporina 87/312 violan la regla del peso molecular y cumplen con los otros parámetros que los consideran aceptables.

Las demás propiedades que aparecen en la tabla 4 corresponden al número de enlaces rotables y el área de superficie polar topológica (TPSA), el primero es una medida de flexibilidad molecular, mientras que el segundo es un descriptor de la absorción intestinal, el potencial de enlace de hidrógeno, biodisponibilidad, entre otras; ambos son utilizados en la regla de Veber y contribuyen en la predicción de la biodisponibilidad oral de una molécula. Según la investigación de Veber y su equipo, las moléculas que tienen 10 o menos enlaces rotables en su estructura y un TPSA igual o inferior a $140 \AA^{2}$ están asociadas a una buena biodisponibilidad oral (Veber et al., 2002); debido a que ninguno de los compuestos reportado presentó un valor de TPSA inferior al establecido y algunos tienen pesos moleculares superiores a $500 \mathrm{~g} / \mathrm{mol}$, es posible que estas moléculas no posean una biodisponibilidad oral aceptable.

\section{Conclusiones}

Después de realizados los cálculos anteriormente descritos y analizados los resultados, se puede concluir que con la información disponible en base de datos como PubChem, se puede utilizar para realizar estudios de relación estructura - actividad para encontrar potenciales moléculas con mayor afinidad por la transpeptidasa y menos afinidad por las betalactamasa (efecto antibiótico dual). La realización de sustituciones químicas en las estructuras de los potenciales antibióticos varían los valores de afinidad con respecto a las enzimas estudiadas, provocando que el efecto antibiótico se vea potenciado o 
disminuido. La molécula cefaperozona monobactam sustituida con un grupo metilo mejoró la afinidad por la transpeptidasa (de -6.8 a $-7.8 \mathrm{kcal} / \mathrm{mol}$ ) y disminuyó la afinidad por la betalactamasa $(-5.9$ a $-5.8 \mathrm{kcal} / \mathrm{mol})$ lo cual está de acuerdo con el objetivo de este trabajo, buscando como fin proponer mejores moléculas antibióticas con menores problemas de resistencia. $\mathrm{Al}$ sustituir la molécula de cefalosporina $87 / 312$ con un grupo metilo, aumentó significativamente la afinidad por la betalactamasa, demostrando un efecto contrario al buscado en la investigación; adicionalmente al aplicar las predicciones ADME a las moléculas modificadas, se encontró que no cumplen con las reglas de Veber sobre la biodisponibilidad. Los resultados presentados en este trabajo sirven como plataforma para la creación de nuevos compuestos con actividad dual sobre las enzimas transpeptidasa y betalactamasa, mediante la sustitución con un mayor número de grupos funcionales que permitan un diseño racional de fármacos, con el fin de validar un mayor número de compuestos y proponer estructuras moleculares con las características antibióticas duales.

\section{Referencias}

Abreu. S.. Varela. Y.. Millán. B.. \& Araque. M. (2014). Klebsiella pneumoniae y Escherichia coli productoras de beta-lactamasas de espectro extendido, aisladas en pacientes con infección asociada a los cuidados de la salud en un hospital universitario. Enfermedades Infecciosas y Microbiología, 34(3), 92-99.

Acar, A., Karaahmetoğlu, G., Akalın, H., \& Altay, A. F. (2019). Pooled prevalence and trends of antimicrobial resistance in Pseudomonas aeruginosa clinical isolates over the past 10 years in Turkey: a metaanalysis. Journal of global antimicrobial resistance, 18 , 64-70.

Acevedo-Cepeda D, Chaparro-Lozano D, Serrato-Ladino KN, Rincón-Riveros A. (2021) Nuevos inhibidores de betalactamasas, actualidad y aplicación en la práctica.Hechos Microbiol. 12(1):41-55.

Ahumedo-Monterrosa, M., Galindo, J. F., Lorduy, J. V., AlíTorres, J., \& Vivas-Reyes, R. (2019). The role of LasR active site amino acids in the interaction with the Acyl Homoserine Lactones (AHLs) analogues: A computational study. Journal of Molecular Graphics and Modelling, 86, 113-124.
Bjerrum, E. J. (2016). Machine learning optimization of cross docking accuracy. Computational biology and chemistry, 62, 133-144.

Centro de prensa OMS. (30 de Abril de 2014). El primer informe mundial de la OMS sobre la resistencia a los antibióticos pone de manifiesto una grave amenaza para la salud pública en todo el mundo. Obtenido de: http://www.who.int/mediacentre/news/releases/2014/a mr-report/es/.

Daina, A., Michielin, O., \& Zoete, V. (2017). SwissADME: a free web tool to evaluate pharmacokinetics, druglikeness and medicinal chemistry friendliness of small molecules. Scientific reports, 7(1), 1-13.

Dennington, R., Keith, T., \& Millam, J. (2009). Semichem Inc., Shawnee Mission Ks. GaussView, Version, 5.

García, C., Astocondor, L., \& Banda, C. (2012). Enterobacterias productoras de-lactamasas de espectro extendido: Situación en América Latina y en el Perú. Acta médica peruana, 29(3), 163-169.

García-Hernández, A. M., García-Vázquez, E., HernándezTorres, A., Ruiz, J., Yagüe, G., Herrero, J. A., \& Gómez, J. (2011). Bacteriemias por Escherichia coli productor de betalactamasas de espectro extendido (BLEE): significación clínica y perspectivas actuales. Revista

española de quimioterapia, 24(2).

Hernández-Gómez, C., Blanco, V. M., Motoa, G., Correa, A., Vallejo, M., \& Villegas, M. V. (2014). Evolución de la resistencia antimicrobiana de bacilos Gram negativos en unidades de cuidados intensivos en Colombia. Biomédica, 34, 91-100.

Hincapié PA, García JL, Gómez D, Mejía L, Holguín A, Uribe $\mathrm{PA}$, et al. Reacciones adversas a betalactámicos: una revisión de tema. Med UPB. 2021;40(1):55-64.

Kumar. G. P.. Sharmila. J. S.. \& Murugan. S. (2014) Docking of CTX-M-9 group of enzymes with drugs and inhibitors and their evolutionary relationship. Asian Journal of Pharmaceutical Clinical Research, 7(1), 237-242.

Kapoor, Y., \& Kumar, K. (2019). Quantitative structure activity relationship in drug design: An overview. SF Journal of Pharmaceutical and Analytical Chemistry, 2, 1017.

Mahasenan, K. V., Molina, R., Bouley, R., Batuecas, M. T., Fisher, J. F., Hermoso, J. A., ... \& Mobashery, S. (2017). Conformational dynamics in penicillin-binding protein $2 a$ of methicillin-resistant Staphylococcus aureus, allosteric communication network and enablement of catalysis. Journal of the American Chemical Society, 139(5), 2102-2110. 
Mendoza-Huizar, L. H. (2018). Reactividad química de la insulina 2HIU empleando la función Fukui. Afinidad, 75(584).

Morris. G. M.. Huey. R.. Lindstrom. W.. Sanner. M. F.. Belew. R. K.. Goodsell. D. S.. \& Olson. A. J. (2009) AutoDock4 and AutoDockTools4: Automated docking with selective receptor flexibility. Journal of Computational Chemistry, 30(16): 2785-2791.

Morris. G. M.. Goodsell. D. S.. Halliday. R. S.. Huey. R.. Hart. W. E.. Belew. R. K.. \& Olson. A. J. (1998) Automated docking using a Lamarckian genetic algorithm and an empirical binding free energy function. Journal of Computational Chemistry, 19(14): 1639-1662.

Owen. L.. White. A.. \& Laird. K. (2017) BioautographyGuided Identification of Antimicrobial Essential Oil Components of Oregano. Cumin and Rosewood against Antibiotic Sensitive and Resistant Staphylococcus aureus. Enterococcus faecium. Escherichia coli and Pseudomonas aeruginosa. American Society of Microbiology. General Meeting. 2017.

Pagadala, N. S., Syed, K., \& Tuszynski, J. (2017). Software for molecular docking: a review. Biophysical reviews, 9(2), 91-102.

Red Nacional de la Vigilancia de la resistencia Bacteriana y de las Infecciones Asociadas al Cuidado de la salud. \& (Colombia). Estado del Arte de la Resistencia Bacteriana y la Vigilancia Epidemiológica de las
Infecciones Asociadas al Cuidado de la Salud En Colombia. 2010; 1-31.

Santos. V. L. D. A.. Gonsalves. A. D. A.. \& Araújo. C. R. M. (2018) Abordagem didática para o desenvolvimento de moléculas bioativas: regra dos cinco de lipinski e preparação de heterociclo 1. 3. 4-oxadiazol em forno de micro-ondas doméstico. Quimica Nova, 41(1): 110115.

Urbina-Daza, A., Anaya-Ortega, C., Ahumedo-Monterrosa, M., Gaitan-lbarra, R., \& Anaya-Gil, J. (2019). Estudio teórico de compuestos derivados de cefalosporinas con potencial actividad inhibitoria frente a proteína de unión a penicilina (PBP) y resistencia a enzimas betalactamasa de espectro extendido (BLEE) producidas por Escherichia coli. Revista Colombiana de Ciencias Químico-Farmacéuticas, 48(1), 44-60.

Veber, D. F., Johnson, S. R., Cheng, H. Y., Smith, B. R., Ward, K. W., \& Kopple, K. D. (2002). Molecular properties that influence the oral bioavailability of drug candidates. Journal of medicinal chemistry, 45(12), 2615-2623.

Yıldırım, H., Bayrak, N., Tuyun, A. F., Kara, E. M., Çelik, B. Ö., \& Gupta, G. K. (2017). 2, 3-Disubstituted-1, 4naphthoquinones containing an arylamine with trifluoromethyl group: synthesis, biological evaluation, and computational study. RSC advances, 7(41), 25753-25764. 